\title{
Analysis of KrF excimer laser beam modification resulting from ablation under closed thick film flowing filtered water.
}

\author{
Colin Dowding \\ Jonathan Lawrence \\ Wolfson School of Mechanical and Manufacturing Engineering, Loughborough \\ University, Loughborough, Leicestershire. LE11 3TU. United Kingdom.
}

\section{Correspondence}

Mr. Colin Dowding

Wolfson School of Mechanical and Manufacturing Engineering,

Loughborough University,

Leicestershire,

LE11 3TU,

Great Britain.

Tel : +44 (0)1509 227593

Fax : +44 (0)1509 227648

e-mail : c.f.dowding@lboro.ac.uk 


\section{Abstract:}

The application of closed thick film liquid to immerse the ablation etching mechanism of an excimer laser poses interesting possibilities concerning debris control, modification of machined feature topography and modification of ablation rate. Further more, these parameters have been shown to be dependant on flow velocity; hence offering further user control of machining characteristics. However the impact of this technique requires investigation. This contribution offers comparison of the calculated ablation pressure and the effect on feature surface characteristics given for laser ablation of bisphenol A polycarbonate using $\mathrm{KrF}$ excimer laser radiation in ambient air against laser ablation of the same substrate under closed thick film flowing filtered water immersion. Also, the impact of such immersion equipment on the optical performance of the micromachining centre used is quantified and reviewed. The pressure is calculated to have risen some $53 \%$ when using the liquid immersed ablation technique. This increase in pressure is proposed to have promoted the frequency of surface Plasmons and asperities with a surface area less than $16 \mu \mathrm{m}^{2}$. The focal length of the optical system was accurately predicted to be increased by $2.958 \mathrm{~mm}$ when using the equipment composed of a $5 \mathrm{~mm}$ thick ultraviolet grade fused silica window covering a 1.5 $\mathrm{mm}$ thick film of filtered water flowing at $1.85 \mathrm{~m} / \mathrm{s}$. This equipment was predicted to have increased the optical depth of focus via reduction in the angle of convergence of the two defining image rays, yet the perceived focus, measured by mean feature wall angle as a discrete indication, was found to be $25 \%$ smaller when using the closed thick film flowing filtered water immersion technique than when laser ablating in ambient air. A compressed plume interaction is proposed as a contributing factor in this change. 


\section{Introduction}

Laser ablation is key to growth in the micro and nano manufacturing industries [1-3]. Technology has been continuously refined and improved in terms of laser fluence, optical resolution and production speed since the emergence of the technique in the late 1970's [3]. But at present, progress is held in check by laser ablation generated debris [4-8]. This is because the capability of laser manufacturing techniques has increased to a level where tooling performance is limited not by the repeatability and resolution of the machining method, but by the deleterious effects of the debris created during the process.

Obvious benefits would accrue in terms of the quality of existing products and for current manufacturing costs, as well as to ensure new product development and economic growth, to remove debris as it is generated and therefore preclude the possibility of the debris causing problems. This is termed an 'in-line' methodology and a number of workers have attempted to develop effective systems, which include liquid based techniques. A technique for delivering a long wavelength laser radiation to a workpiece by use of a fine jet of water demonstrate that the use of such mediums does not pose unacceptable attenuation of the beam [9]. The existence and efficacy of ablation plume etching can be proven by the example of laser induced backside wet etching (LIBWE), pioneered by Wang et al. [10]. This process makes laser machining of laser wavelength transparent materials viable by passing the beam through the material to be machined and focusing the beam to a point just beyond the far surface of the material in a liquid medium. The ablation plume generated in the liquid medium attacks and etches the material. Moreover, the use of LIBWE has been found by others to provide an increased ablation rate when compared to that achieved on the same materials by direct machining in ambient air [11-13]. The technique projecting a beam through a liquid medium and onto the surface is less well characterized. A liquid jetting system has been developed and patented [14], but little has been published detailing its 
performance as a technique. The technique of immersing the sample beneath a liquid film that the laser beam traverses to focus on the sample surface has been documented in the effort to produce and collect micro- and nano-particles efficiently [15].

Major inroads have been made by Dowding and Lawrence [16-19] to develop a workable inline debris removal system based on ablating under both open and closed thick film flowing water. The introduction of a liquid to an ablation event poses a significant difference to the typical gaseous of vacuum environments used for ablation machining. The mechanism of ablation in liquids has some significant differences with ablation in gases or a vacuum. The most important of these is that the liquid confines the expansion and movement of the plasma plume due to its higher density and viscosity. This confinement can greatly influence the thermodynamic and kinetic properties of the plasma and therefore the evolution of the plume. This can give rise to distinctly different scenarios both during vaporization and condensation compared to ablation in gaseous or vacuum environments [20].

The impact of the equipment developed by Dowding and Lawrence [17-19] and the techniques it facilitates on the broader machining parameters involved in laser micromachining. This contribution describes fundamental changes to the techniques involved in laser machining with respect to laser machining in ambient air: ablation plume pressure; optical focal length; and depth of focus.

\section{Experimental Procedures}

\section{$\underline{\text { Material Details }}$}

Bisphenol A polycarbonate (Holbourne Plastics, Ltd), was as received in $1200 \mathrm{x} 1000 \mathrm{~mm}^{2}$ sheets of $0.5 \mathrm{~mm}$ thickness. Prior to excimer laser processing, the bisphenol A polycarbonate sheet was cut into rectangular sections of $8 \times 12 \mathrm{~mm}^{2}$ using scissors - a shear cutting 
technique which avoids production of debris. Protective cover sheets were then peeled off each sample.

\section{Laser Details and Experimental Set-Up.}

For both closed immersion and ambient air processing, an excimer laser (LPX200; Lambda Physique, $\mathrm{GmbH}$ ) using $\mathrm{KrF}$ as the excitation medium was used to produce a beam with a wavelength of $248 \mathrm{~nm}$ over $25 \mathrm{~ns}$ pulses. Thereafter, the beam was supplied to a laser micromachining centre (M8000; Exitech, Ltd), where it was passed through a stainless steel mask to produce a $201 \times 203 \mu \mathrm{m}^{2}$ rectangular image. The masked beam was then demagnified through a 4x optic (Francis Goodhall, Ltd) to produce an ablation spot with a depth of focus $(\mathrm{DoF})$ of $6 \mu \mathrm{m}$. A profile of the masked beam was obtained using a beam profiler (SP620U; Spiricon, Ltd), which showed that the beam shape had an even distribution, with only a slight positive skew across the y-axis; demonstrating good positioning of the mask in the raw beam.

Focus was found by narrowing the focal rage until satisfactory focus was achieved. Pulse energy was measured out of focus, using a power meter head (J50LP-2; Molectron Detector, Inc.) connected to a reader unit (Energy Max 400; Molectron Detector, Inc.). Spot energies were measured six times for each sample - three times before the sample was machined and three readings were taken after the sample was machined. Each reading was taken after the system attenuator had been reset. In this way any change in the beam between measurements and any inaccuracy in the positioning of the attenuator were accounted for.

Ten sites were machined on all samples using six pulses for each feature. These were produced at regular, consecutive focal lengths, given in Table 1. In this instance the beam was attenuated by a tool attenuator set at $126^{\circ}$ from minimum transmission, resulting in a beam fluence of $590.4 \mathrm{~mJ} / \mathrm{cm}^{2} \pm 9.2 \mathrm{~mJ} / \mathrm{cm}^{2}$ for ablation in ambient air and under filtered water immersion that produced features of $203 \times 205 \mu \mathrm{m}^{2}$. This fluence data was calculated 
from pulse energy data taken using a pulse energy head (J50LP-2; Molectron Detector, Inc.) positioned above the focal point of the laser, and enumerated by a laser energy meter (Energy Max 400; Molectron Detector, Inc.). The fluence was calculated using the mean beam energy measured (averaging techniques were employed for experimental rigour: beam pulse energy was recorded five times before and after machining each sample, every value recorded was the mean value measured over 100 pulses - between readings the attenuator was reset to account for attenuator position errors) so that any changes in laser output over time were accounted for.

\section{Ambient Air Laser Processing}

Samples machined in ambient air were produced, using the same laser and micromachining equipment as the closed immersion ablation samples. The bisphenol A polycarbonate samples were mounted directly to the vacuum chuck inside the micromachining station (M8000; Exitech, Ltd). After lasing ended the sample was removed and placed into the cell of a sealed sample tray to protect them from atmospheric dust.

\section{Closed Thick Film Filtered Water Immersion Laser Processing Procedure}

Figure 1(a) describes the critical experimental layout of the sample once clamped inside the flow rig, which was mounted to the side of the sample vacuum chuck of the laser microprocessing centre (M8000; Exitech, Ltd.). The sample was positioned in the centre of the flat aluminium table between the water supply and exit holes. The sample was retained by a recess in a spacer plate that lay in contact with the aluminium sample table. An O-ring cord, located by a rectangular groove in the sample table, provided a seal between the sample table and the spacer plate. On the top of the spacer plate a second oval O-ring was housed in another machined groove. This acted as a gasket between the spacer plate and the beam window - a $25 \times 25 \times 5 \mathrm{~mm}^{3}$ ultra-violet grade fused silica sheet (Comar Instruments, Ltd). 
The beam window was retained by a diamond shaped recess in a third aluminium plate, $8 \mathrm{~mm}$ in thickness to provide stiffness to the whole sandwich.

Figure 1(b) shows the water filtering and supply system. Water originated from normal mains supply by wall tap. The water was poured into a domestic water filter (Britta, Inc.) situated at the top the water supply assembly to remove typical corrosive elements present in mains water. The water was then retained in a header tank located above the pump and, under the action of gravity, was forced into the $700 \mathrm{~W}$ pump chamber (CPE100P, Clarke Power Products, Ltd.). The pump forced the water through a water flow rate meter (FR4500, Key Instruments, Inc.) and then along a $3 \mathrm{~m}$ distance through a $6 \mathrm{~mm}$ outer diameter nylon tube to the inlet push-in elbow fitting on the bottom of the sample table. Last, the water was returned along a further $3 \mathrm{~m}$ through a $6 \mathrm{~mm}$ outer diameter nylon tube to a collection bucket. The pump was capable of producing 4.2 bar at the outlet, equating to a maximum flow rate through the ablation chamber of $3.89 \mathrm{~m} / \mathrm{s}$, given losses along the supply and return tubing. Precise control of the flow rate was provided by a variable valve of the flow-meter. For this work, a flow velocity of $1.85 \mathrm{~m} / \mathrm{s}$ was used for this work.

\section{$\underline{\text { Sample Analysis Techniques }}$}

Two methods can be used to measure and demonstrate the focal range of an optical system. The simplest technique is that of micrograph inspection. This allows identification of features that appear to have ill defined edges. Figure $2 \mathrm{a}$ shows a feature that appears sharp, Figure $2 \mathrm{~b}$ a figure that is slightly out of focus and Figure $2 \mathrm{c}$ a feature that is a long distance from the ideal focal position.

Another, more quantative method than micrograph inspection is the adoption of inductive needle profiling. Using this technique, the cross sectional profiles of features can be obtained, allowing more detailed analysis of feature wall angle and sample floor profile. The samples were placed under a needle profiler (Talysurf 300; Taylor-Hobson, Ltd.). Software 
(Mountains; Taylor-Hobson, Ltd) was used to process and interpret the raw data generated. Profiles of the features imaged in Figure 2 are given alongside for direct comparison. This demonstrates the possibility of this technique for discreet quantative analysis of the focus of the light at a specific focal length.

\section{Results and Discussion}

\section{Modification of the ablation pressure: a cause for feature floor darkening}

It is sensible to consider PLA in gaseous media first. Three distinct phases have been identified to occur during such an interaction. The initial phase of the pulse will instigate ablation, and is termed 'generation': the gaseous materials ejected from the target will form a plasma as depicted in Figure 3(a). The plasma formed will be partially confined by the surrounding gaseous environment; however continued ablation and excitation of the plume will cause the plume to expand, as shown in Figure 3(b) [15].

The ablated species originating from the target material are not suspended in the plasma plume. Because both the plasma and the surrounding environment are gaseous in nature, the two tend to mix. The high energy and temperature state of the plume allows chemical reactions to occur between molecules from the two substances to form new compounds, this chemical modification is termed 'transformation.'. The latter part of the pulse adds energy to instigate these reactions; the mixing of the gas and plume continues after the pulse has ended as the next phase begins, as described in Figure 3(c) [15].

The heat contained within the plume begins to diffuse into the surrounding environment at the end of the pulse. The intense thermal diffusion of the species in the plasma plume can result in collisions, leading to aggregations. Thus thermal diffusion is also critical in transformation, before eventually leading to 'condensation' of the new mixed species into melt form and finally to solidification resulting in particulate matter [15]. 
Fabbro et al. [21] found that a shockwave was induced by the laser induced plasma and was confined to the extents of the plume by the surrounding liquid (see Figure 4). The laser induced plasma adiabatically expands when the plasma absorbs the latter part of the laser pulse and receives a further supply of vaporized mass from the target material at a supersonic velocity to create a shockwave as described in Figure 4(b). This shockwave will generate increased pressure within the laser induced plasma plume. This increase in pressure generated by the shockwave has become known as the 'plasma induced pressure'. Thermodynamically, the increase in pressure will be coupled to an increase in temperature of the plasma, causing the laser induced plasma under the confinement of the surrounding liquid to have a higher temperature, pressure and density than that of a plasma generated in a gaseous or vacuum environment. The plasma induced pressure is dependant upon the wavelength and fluence of the beam involved [22], with the maximum pressure achievable decreasing with increasing pulse length [23].

Fabbro has been involved in a wide range of research to understand the nature of the plasma induced in a solid-liquid system [21, 23, 24]. Fabbro and colleagues have produced an analytical model to predict the maximum pressure, $\mathrm{p}_{\max }$, measured in GPa, generated by the laser induced plasma in water:

$$
p_{\max }=0.01 \sqrt{\frac{\Omega}{\Omega+3}} \sqrt{Z} \sqrt{P_{i}},
$$

where $\Omega$ is the fraction of internal energy released as thermal energy (typically $\Omega \approx 0.25$ ), $\mathrm{P}_{\mathrm{i}}$, is the incident power intensity and $\mathrm{Z}$ is the reduced shock impedance between the target and the confining liquid (filtered water), defined as

$$
\frac{2}{Z}=\frac{1}{Z_{\text {water }}}+\frac{1}{Z_{t \arg e t}},
$$

where $Z_{\text {air }}, Z_{\text {water }}$ and $Z_{\text {target }}$ are the shock impedances of air, water and the target respectively; these values are identical to those commonly quoted as acoustic impedance values [24], 
measured in Rayles $\left(\mathrm{N} / \mathrm{s} \cdot \mathrm{m}^{3}\right)$. The acoustic impedances for the mediums and material used in this target have been collected [25]: air has an impedance of 413.2 Rayles; water 1480 Rayles and Polycarbonate is larger still with 2770 Rayles. Calculation using the values given above and Equations (1) and (2), allows the estimation of the plume pressure during the laser pulse. The pressure generated in ambient air is predicted to be an increase of $7.9 \mathrm{GPa}$, a large increase over atmospheric pressure; however, the use of filtered water immersion posed a significantly larger increase in plume pressure of $12.1 \mathrm{GPa}$, a result that is a multiple of 1.6 greater than the pressure generated in a similar plume in ambient air.

The change in plume pressure and the resultant increase in plume temperature, coupled with greater plume density, which had the effect of increasing plume attenuation contributions when machining using closed thick film filtered water immersion of the sample may impact the machining mechanism in several significant manners. The result of this is evident in the darkened surface of the all the samples machined. Cleaning of these samples did not result in removal of this blackened surface as the two features given in Figure 5 show: Figure 5(a) gives a sample after machining without any post-process cleaning, clearly displaying the characteristic darkened surface attribute of closed thick film filtered water immersed laser machining; Figure 5(b) shows the sample feature following 10 minutes ultrasonic cleaning in DI water.

The apparent change in medium chemistry could be a result of charring of the feature surface. This charring could have been promoted by increased plume temperature which was thermodynamically coupled with the calculated increased plume pressure. However, the lack of impact caused by ultrasonic bath immersion of 10 minutes in ultrapure water on the feature shown in Figure 5 suggests that this is not a significant contributor in this case, as such a rigorous cleaning technique should have removed oxidised detritus from the feature surface leaving an apparently brighter surface. 
Alternately, the darkening could be the result of a number of scenarios. Previous research has identified the existence of laser ablation generated and controllable surface plasmon features on the surfaces of nanoparticles manufactured using laser ablation [26, 27]. The quaziparticle offers a method of specifying a magnitude of the cumulative plasma oscillation existent in the free electron gas of any substance [26]. A surface plasmon is a more specific item, that occurs only on the outer electron shells of exposed atoms of surfaces. These interact strongly with light, causing significant polarization of planar light, it is these that produce the anomalies recorded in diffraction of light from metal surfaces [28]. Surface plasmons can have critical modification of the reflected electromagnetic radiation; they have been used to modify the apparent colour of surfaces and can afford the ability for low optical diffraction levels below the traditional optical diffraction limit [29]. Both of these effects can go some way to explain the observed darkening of the features machined under closed thick film flowing filtered water immersion. Some previous contributions have proposed that plasmon production and size is proportional to laser ablation plume pressure [26], thus the pressure increase calculated above for the techniques described in this contribution could result in significant modification to the interaction between light and the surface of machined features.

Additionally, the modification of the ablation mechanism, to feature a significant plume etching contribution as alluded to in previous contributions [18, 19, 30], where the drag of immersion fluid flowing past the ablation plume distorted the plume geometry. Varying the flow velocity resulted in modified ablation threshold [18], ablation rate [30] and surface topography [19]. The recorded modification of surface topography demonstrated that the surface roughness was proportional to flow velocity and laser fluence. It also demonstrated a significant increase in surface roughness of the features. This increase in surface roughness would have the effect of high diffusivity of light supplied from the microscope objective, reducing the intensity of light reflected to the camera as described schematically in Figure 6. 
Such a characteristic could doubtlessly have a significant use in the displays or lighting industry, where even light distribution over a broad area is a desirable feature.

\section{Modification to the focal length of the optical system.}

A simplistic prediction of the change in focal position of the laser system could be calculated before any machining was conducted, using the technique of ray tracing [31]. For a true calculation of the beam characteristics, thin lens theory states that a number of dimensions must be known for an imaging system such as that used in an Excimer laser micromachining tool, as illustrated in Figure 7. The system described in Figure 7 is a simple case where the beam does not experience any change in medium. The system involved in the ablation of a substrate immersed in a closed thick film of filtered water is much more complex, requiring the beam to pass from the demagnifying optic, into the ambient air of the system, on to enter the substrate immersion apparatus fluid enclosing window before finally leaving the window and traversing the immersion fluid itself. The changing refractive index will have the effect of modifying each ray as described in Figure 7. If the angle of incidence of a ray, $\theta_{\mathrm{i}}$, is known, the refraction at each stage can be calculated rearranging the definition of the refractive index to give:

$$
\theta_{r}=\sin ^{-1}\left[\frac{\sin \theta_{i}}{n}\right]
$$

to give the angle of refraction of the same ray. The maximum angle of incidence, can be found when the distance between the beam and the substrate and the change in radius of the beam are known. The tool used in this contribution had a lens - substrate separation of approximately $65 \mathrm{~mm}$ (calculated to be $63.438 \mathrm{~mm}$ using thin lens theory); the radius of a planar wave passing the edge of the mask orifice was $400 \mu \mathrm{m}$ and then went on to pass through the edge of the lens and being demagnified to the focal point where the image had a 
radius of $102 \mu \mathrm{m}$, giving a maximum angle of incidence, denoted as $\theta_{\mathrm{ia}}$ and described by line ' $\mathrm{I}_{\mathrm{a}}$ ' in Figure 7, of $0.45^{\circ}$.

The other image defining ray passes from the edge of the mask, through the centre of the lens and onto the opposite edge of the image at the feature, labelled as ' $\mathrm{I}_{\mathrm{b}}$ ' in Figure 7 which will now be denoted $\theta_{\mathrm{ib}}$. In the case of the tool used for this work, the distance between the mask and the substrate was $250 \mathrm{~mm}$, thus knowing the dimensions of the mask, feature and the separation of lens and substrate as stated above, gives an angle of incidence of $0.09^{\circ}$ for $\theta_{\mathrm{ib}}$. When a ray passes from one medium to another, the relationship between the refractive indices of both mediums must be taken into account. This is done by application of Snell's law [32], which can be written as

$$
\theta_{r}=\sin ^{-1}\left[\frac{n_{1} \sin \theta_{i}}{n_{2}}\right]
$$

where $\mathrm{n}_{1}$ is the refractive index of the medium being passed from and $\mathrm{n}_{2}$ is the medium being passed to.

Using Snell's Law, it can be calculated that a ray passing from the medium of air surrounding the micromachining centre, that possesses a refractive index value of 1.0008 for electromagnetic radiation with a wavelength of $248 \mathrm{~nm}$ [33], into the ultra violet (UV) grade fused silica, which possessed a measured refractive index of 1.5084 at the same wavelength [34], resulted in the modification to the path vectors of $I_{a}$ and $I_{b}$. As the light passed through the fused silica window the angle of refraction of $\mathrm{I}_{\mathrm{a}}, \theta_{\text {ra }}$, was $0.3^{\circ}$, and the angle of refraction of $\mathrm{I}_{\mathrm{b}}$ was $0.06^{\circ}$.

As one can see in Figure 8, the angle of the refraction at the first medium interface becomes the angle of incidence for the second, denoted as $\theta_{\text {ii }}$ in Figure 8. Again, Snell's Law can be used here to calculate the final range of angles of refraction, $\theta_{\mathrm{rr}}$, of any rays including or between $I_{a}$ and $I_{b}$ in Figure 7. The reactive index of filtered water on $248 \mathrm{~nm}$ wavelength 
electromagnetic radiation is 1.3787 [35]. Knowing this, and assuming the boundary between the water and the fused silica window is regular and without cavity, the angles of refraction, $\theta_{\text {rra }}$ and $\theta_{\text {rrb }}$ are derived to be $0.3^{\circ}$ and $0.07^{\circ}$ respectively.

Given these angles of modification and knowing the micromachining system was left unchanged as the laser machining was moved from ambient air to having the liquid immersion equipment mounted and filled with flowing filtered water, the change in focal length of the optical system can be calculated using successive application of simple trigonometry. The thick film flowing immersion equipment used for this contribution was composed of $1.5 \mathrm{~mm}$ of filtered water covered by $5 \mathrm{~mm}$ UV grade fused silica. Knowing these dimensions allows the calculation of the modification to the focal length of the system, denoted $\Delta 1$ in Figure 8 , which was predicted to be a $2.958 \mathrm{~mm}$ increase.

In reality, the immersion rig sample mounting surface was mounted $1.7 \mathrm{~mm}$ beneath the level of the vacuum chuck, The two features, machined in ambient air and under thin film flowing filtered water immersion with optimum focus are indicated by a square in Figure 9(a) and Figure 9(b) respectively. The stage height of the lowest sample in Figure 9(a) was at 3.475 $\mathrm{mm}$ from it's home position. The stage elevation between each feature was $0.025 \mathrm{~mm}$, meaning that the focal position for machining in ambient air was found at $3.675 \mathrm{~mm}$ from the home position of the stage. For the closed thick film filtered water immersed samples, shown in Figure 9(b) the lowest feature height was $2.275 \mathrm{~mm}$ from the homing position, meaning the focal position was found in this case to be $2.475 \mathrm{~mm}$. This gives an increased focal length by relative stage position of $1.2 \mathrm{~mm}$ for the immersed sample over that of the sample machined in ambient air. Coupling this value with the $1.7 \mathrm{~mm}$ deviation in mounting height results in a $2.9 \mathrm{~mm}$ increase in focal length, a value very close to that predicted mathematically above. This result means that the modification of beam path can be easily 
predicted given known equipment dimensions, when using closed thick film flowing immersed laser ablation machining techniques.

\section{Modification to the depth of focus}

The depth of focus is the tolerance in the focal length of the optical system used to guide a laser beam from the source, through the objective, through the demagnifying lens and onto the image. The depth of focus is defined primarily by the characteristics of the demagnifying lens; but, the application of further optics may modify this dimension. Moreover, modifying the ablation mechanism from one that is dependant upon the laser etching provided by a beam in focus, to one that can operate using the etching contribution of a compressed plume by use of a compressed ablation plume, as has been proposed previously [17-19, 30], could present the possibility of an increased range of focal length that produces an acceptable image that can be deemed to be 'in focus'. Alternatively, the implication of such a mechanism could equally reduce the range of focal length that produces an equivalent feature definition to that in ambient air.

Figure 10 plots the average wall angle, taken from 10 profiles of features machined at each focal length, with respect to 10 focal displacements, separated by increments of $0.05 \mathrm{~mm}$. Two plots are given, a plot describing the angle between level and the wall of ten features machined in ambient air and for a feature machined under closed thick film flowing filtered water ablation at intervals of $0.1 \mathrm{~mm}$ in focal length. These results show that use of immersion can produce wall angles equivalent to those achieved by laser machining in ambient air; however, the range of focal length that high wall angles were produced in was smaller when using laser ablation under closed laser ablation immersion. This result indicates that the depth of focus is reduced. Interpolation of the polynomial best fit given for each group of data identifies that a wall angle greater than $77^{\circ}$ was achieved by ambient air over a 
range of $0.2 \mathrm{~mm}$. For the samples machined using laser ablation under closed thick film flowing filtered water immersion, this range was 0.15 . This indicates a reduction in the depth of focus of the system by $25 \%$ when using immersion. This result cannot be the result of an optical change to the beam by the immersing fluid and associated equipment; the installation of this apparatus has the effect of reducing the separation of the rays $\mathrm{I}_{\mathrm{a}}$ and $\mathrm{I}_{\mathrm{b}}$, as denoted in Figure 7, where the beam was $3 \mathrm{~mm}$ above the surface of the material. Hence the actual depth of focus was marginally increased as reduced divergence between the rays was afforded. Given this knowledge, it is clear that the modification to the ablation plume by use of closed thick film flowing filtered water ablation results in reduced feature definition, when using the flow velocity of $1.85 \mathrm{~m} / \mathrm{s}$ as used for this work.

\section{Conclusions}

Comparison of the calculated ablation pressure and the effect on feature surface characteristics is given for laser ablation of bisphenol A polycarbonate in ambient against the laser ablation of the same substrate under closed thick film flowing filtered water immersion. Also, the impact of such immersion equipment on the optical performance of the micromachining centre used is quantified and reviewed.

The characteristics of pulsed laser ablation in gaseous mediums, such as air, are known and well documented. This occurred in four phases; three of these are of interest for laser ablation machining of features in materials. Interaction of the laser beam with the surface results in 'generation' of a laser ablation plume. This plume then absorbed a proportion of laser energy by various contribution plume attenuation effects, resulting in plume heating and rapid volumetric expansion of the plume, which was resisted little by the surrounding gaseous medium. Thermal diffusion then followed and with it came condensation and plume collapse. The lack of constraint to the expansion of the plume results in a plume with short life and low 
internal pressure, calculated to be $7.9 \mathrm{GPa}$, using previously defined equations and the appropriate shockwave impedance values for the materials and medium used.

Immersion of the ablation plume in filtered water resulted in a modification to the ablation mechanism as has been documented previously. The ablation plume was less free to expand once generated by the initial phase of the laser pulse, this restriction in growth was caused by the increased viscosity of the filtered water medium and the vastly larger shock impedance that comes associated. The compression of the ablation plume resulted in a calculated plume pressure of $12.1 \mathrm{GPa}$, an increase of $53 \%$ over the value achieved in ambient air. Increased pressure resulted in associated thermal increase and greater plume density, thus increasing the combined plume attenuation effects - further raising the plume temperature.

Surface oxidation and charring could be the cause for the darkened feature base surfaces observed to be a result of using this equipment; however, this is unlikely after ultrasonic bath immersion in ultra pure water did not remove significant amounts of charred material as would have been expected. Previous contributors have shown a correlation between laser ablation plume pressure and surface Plasmon size and frequency. Since the ablation plume pressure was increased significantly by laser ablation plume confinement when using closed thick film flowing filtered water immersion, this phenomena could be the cause, partially or as contributor thereof; where the darkening is an optical symptom of the plasmons. It is believed the largest contribution was given by the effect of dramatically increased surface roughness, where the amplitude of surface asperities with base areas lower than $16 \mu \mathrm{m}^{2}$, acted to reflect light unevenly from the surface to diffuse the light and prevent it from being reflected back to the observer through the microscope objective. If this was the case, such a characteristic could doubtlessly have a significant use in the displays or lighting industry, where even light distribution over a broad area is a desirable feature. 
The refraction of the laser image by introduction of the closed thick film flowing filtered water immersion was calculated by use of the thin lens theorem, Snell's law and the ray tracing technique. This technique predicted an increase to the focal length of the system of $2.958 \mathrm{~mm}$, given the known thicknesses of 1.5 and $5.0 \mathrm{~mm}$ and refractive indexes for $248 \mathrm{~nm}$ radiation of 1.3787 and 1.5084 of the filtered water and UV grade fused silica respectively, obstructing the beam when the equipment was installed in the ambient air of the micromachining centre used, which typically has a refractive index of 1.0008 . The actual increase in focal length measured to be in best focus when using the closed thick film flowing filtered water immersion equipment was $2.9 \mathrm{~mm}$, showing that this modelling technique is relatively accurate and can be used as a tool for simple and quick system design.

The modification to the depth of focus was measured discretely by comparing the variance in wall angle as samples machined in ambient air and under closed thick film flowing filtered water immersion moved outside the focal tolerance of the demagnifying optic installed in the micromachining centre. This findings of this work indicated that the use of closed thick film flowing filtered water immersion can match the level of focus achieved by laser machining in ambient air. When machining bisphenol A polycarbonate in ambient air, the focal length of the system could vary by $0.1 \mathrm{~mm}$ around the optimum focus to produce a measured mean wall angle of more than $77^{\circ}$; the same wall angle was only achievable over a range of 0.075 $\mathrm{mm}$ when machining under closed thick film flowing filtered water immersion. This indicates a $25 \%$ reduction in depth of focus, that cannot be accredited to beam modification by the equipment, as this actually had the effect of increasing the depth of focus by virtue of reducing the angle of convergence of the characteristic rays in the image. This loss in perceived depth of focus must be due to the modification and proposed increased contribution of plume etching to the ablation mechanism. 


\section{$\underline{\text { References }}$}

1. Rizvi, N. H., Apte, P. “Developments in laser micro-machining techniques", Journal of Materials Processing Technology, 2002, 127, pp.206-210

2. Dyer, P. E. “ Excimer laser polymer ablation: twenty years on", Applied Physics A, 2003, 77, pp.167-173

3. Gower, M. C. "Excimer laser microfabrication and micromachining", Laser Precision Microfabrication, RIKEN Review, 2001, pp.50-56.

4. Braun, A., Zimmer, K., Hösselbarth, B., Meinhardt, J., Bigl, F., Mehnert, R. "Excimer laser micromachining and replication of 3D optical surfaces", Applied Surface Science, 1998, 127-129, pp.911-914

5. Izatt, J. A., Sankey, N. D., Partovi, F., Fitzmaurice, M., Rava, R. P., Itzkan, I., and Feld, M. S. “Ablation of Calcified Biological Tissue Using Pulsed Hydrogen Fluoride Laser Radiation”, IEEE Journal of Quantum Electronics, 1990, 26 (12), pp.22612270.

6. Ghantasala, M. K., Hayes, J. P., Harvey, E. C., and Sood, D. K. "Patterning, electroplating and removal of SU-8 moulds by excimer laser micromachining", Journal of Micromechanics and Microengineering, 2001, 11, pp.133-139.

7. Lankard J. R., and Wolbold, G., "Excimer Laser Ablation of Polyimide in a Manufacturing Facility", Applied Physics A, 1992, 54, pp.355-359

8. Lobo, L. M., Solid Phase By-Products of Laser Material Processing, 2002, Loughborough University.

9. Richerzhagen, B Material shaping device with a laser beam which is injected into a stream of liquid, 1999, WO/1999/056907, Switzerland. 
10. Wang. J., Niino. H., Yabe. A. "Micromachining of transparent materials with superheated liquid generated by multiphotonic absorption of organic molecule" Applied Surface Science, 2000, 154-155, pp.571-576.

11. Wang. J., Niino. H., and Yabe. A. "Micromachining of quartz crystal with excimer lasers by laser-induced backside wet etching" Applied Physics A, 1999, 69 (S1), pp.S271-S273.

12. Zimmer. K., and Böhme. R. "Precise etching of fused silica for micro-optical applications", Applied Surface Science, 2005, 243 (1-4), pp.415-420.

13. Yasui. Y., Niino. H., Kawaguchi. Y., and Yabe. A. "Microetching of fused silica by laser ablation of organic solution with $\mathrm{XeCl}$ excimer laser", Applied Surface Science, 2002, 186 (1-4), pp.552-555.

14. Scaggs, M. J. Method and apparatus for fine liquid spray assisted laser material processing, 2003, WO/2003/028943, USA.

15. Sattari, R., Sajti, C. L., Kahn, S., and Barcikowski, S. "Scale-up of nanoparticle production during laser ablation of ceramics in liquid media" The proceedings of The $27^{\text {th }}$ International Congress on Application of Lasers and Electro-Optics: Laser Materials Processing Section, 2008, pp.49-54.

16. Dowding, C. F., and Lawrence, J. "Use of thin laminar liquid flows above ablation area for control of ejected material during excimer machining", Proceedings of the Institution of Mechanical Engineers Part B: Journal of Engineering Manufacture, 2009, 223 (7), pp.759-775

17. Dowding, C. F., Lawrence, J. "Ablation debris control by means of closed thick film filtered water immersion", Proceedings of the Institution of Mechanical Engineers Part B: Journal of Engineering Manufacture. Accepted for publication 24/09/2009. 
18. Dowding, C. F., Lawrence, J. "Effects of closed immersion filtered water flow velocity on the ablation threshold of bisphenol A polycarbonate during excimer laser machining", Applied Surface Science. Accepted for publication 06/01/2010

19. Dowding, C. F., Lawrence, J. "The impact of medium chemistry to flowing liquid closed immersion ablation of bisphenol A polycarbonate", Lasers In Engineering. Accepted for publication 20/12/2009.

20. Yang, G. W. "Laser ablation in liquids: Applications in the synthesis of nanocrystals", Progress in Materials Science, 2006, 52 (4), pp.648-698.

21. Fabbro, R., Peyre, P., Berthe, L., and Scherpereel, X. L. "Physics and applications of laser-shock processing“, Journal of Laser Applications, 1998, 10 (6), pp.265-269.

22. Munson, B. R., Donald, F. Y., and Theodore, H. O. Fundamentals of Fluid Mechanics, 2002, 4, John Wiley \& Sons Inc., NY.

23. Peyer, P., Berthe, L., Fabbro, R., and Sollier, A. "Experimental determination by PVDF and EMV techniques of shock amplitudes induced by $0.6-3$ ns laser pulses in a confined regime with water", Journal of Applied Physics, 2000, 33, pp.498-503.

24. Berthe, L., Fabbro, R., Peyre, P., and Tollier, L. "Shock waves from a water-confined laser-generated plasma”, Journal of Applied Physics, 1997, 82 (6), pp.2826-2832.

25. Shackelford, J. F., and Alexander, W. CRC Materials Science and Engineering Handbook, 3, 2001, CRC Press.

26. Kabashin, A. V., Meunier, M. "Synthesis of colloidal nanoparticles during femtosecond laser ablation of gold in water", Journal of Applied Physics, 2003, 94, (12), pp.7941-7943.

27. Dolgaeva, S. I., Simakina, A. V., Voronova, V. V., Shafeeva, G. A., and BozonVerdurazpp, F. "Nanoparticles produced by laser ablation of solids in liquid environment", Applied Surface Science, 186, (1-4), 546-551. 
28. Maier, S. A. Plasmonics: Fundamentals and Applications, 2007, 1, Springer, Oxford.

29. Böer, K. W. Survey of Semiconductor Physics, 2002, 1, (2), Wiley, New York.

30. Dowding, C. F., Lawrence, J. "Excimer laser machining of bisphenol A polycarbonate under closed immersion filtered water with varying flow velocities and the effects thereof on etch rate", Proceedings of the Institution of Mechanical Engineers Part B: Journal of Engineering Manufacture. Accepted for publication 20/12/2009.

31. Glassner, A. S. An introduction to ray tracing, 1989, 1, Elsevier Science, Amsterdam.

32. Hecht, E., Zajac, A., Optics, 2001, 4, Pearson Education, Ltd.

33. Shaw M. J., Hooker, C. J., Wilson, D. C. "Measurement of the nonlinear refractive index of air and other gases at 248 nm", Optics communications, 1993, 103, (1-3), pp.153-160.

34. Equipment data sheet supplied with UV grade fused silica windows by Comar Instruments.

35. Liang, W., Huang, Y., Xu, Y., Lee, R. K., and Yariv, A. "Highly sensitive fiber Bragg grating refractive index sensors", Applied physics letters, 2005, 86 (15), pp.86-89. 


\section{$\underline{\text { List of Figures }}$}

Figure 1(a): the closed thick film filtered water immersion ablation assembly: (1) sample; (2) base plate; (3) sample clamp and flow chamber spacer; (4) U.V. grade fused silica window for laser beam; (5) window clamp; (6) clamping bolts that squeeze components together. Figure 1(b): the fluid supply unit: (1) source water; (2) filtering; (3) filtered water storage; (4) centrifugal pump; (5) flow rate control valve; (6) high pressure flow rate controlled filtered water outlet to flow rate ablation chamber.

Figure 2: Three micrographs and corresponding mean surface profiles of features that are: (a) in focus; (b) drifting out of focus; (c) significantly out of focus.

Figure 3: Three stages of PLA in gasses: (a) initial phase of beam creates a small, rapidly expanding plasma plume; (b) further irradiation ablates more material and energises plume, causing further plume expansion; (c) pulse ends, plume quenching period beings.

Figure 4: Four stages of ablation in a confining fluid: (a) Initial period of pulse instigates plasma plume; (b) end of pulse energises plume and plasma induced shockwave both cause plume expansion, confining liquid limits expansion; (c) Four different reactions occurring in the plasma, the liquid and at the interface between the two.

Figure 5(a): a micrograph displaying the dark floor of sample machined under closed thick film filtered water immersion directly after machining; Figure 5(b): a micrograph displaying the dark floor of sample machined under closed thick film filtered water immersion, after the sample cleaned using ultrasonic bathing for 10 minutes. 
Figure 6: A schematic describing the high diffusion of reflected light from a feature surface with high roughness.

Figure 7: A diagram describing the two defining rays, $\mathrm{I}_{\mathrm{a}}$ and $\mathrm{I}_{\mathrm{b}}$, in an imaging system.

Figure 8: A diagram illustrating the modification to the vector of a ray through changing medium, taking into account Snell's Law.

Figure 9(a): a strip of features machined at $0.025 \mathrm{~mm}$ intervals using $\mathrm{KrF}$ excimer laser ablation in ambient air: feature 9 (boxed) is the optimum feature. Figure 9(b): a strip of features machined at $0.025 \mathrm{~mm}$ intervals using $\mathrm{KrF}$ excimer laser ablation under thick film flowing filtered water immersion: feature 9 (boxed) is the optimum feature.

Figure 10: A graph plotting feature wall angle against increase in focal length for features machined by $\mathrm{KrF}$ excimer laser ablation in ambient air and under closed thick film flowing filtered water. 
Figure 1
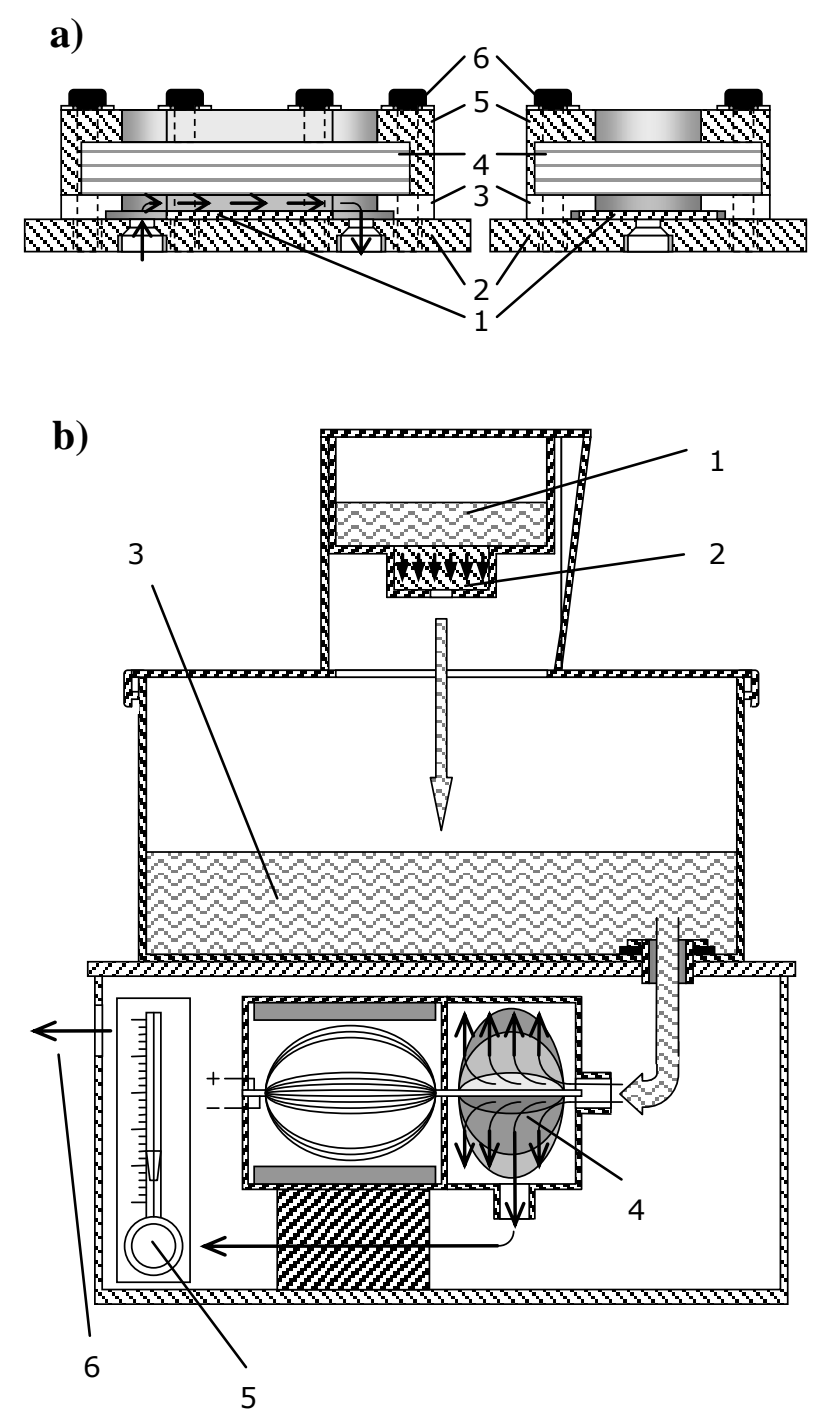
Figure 2:

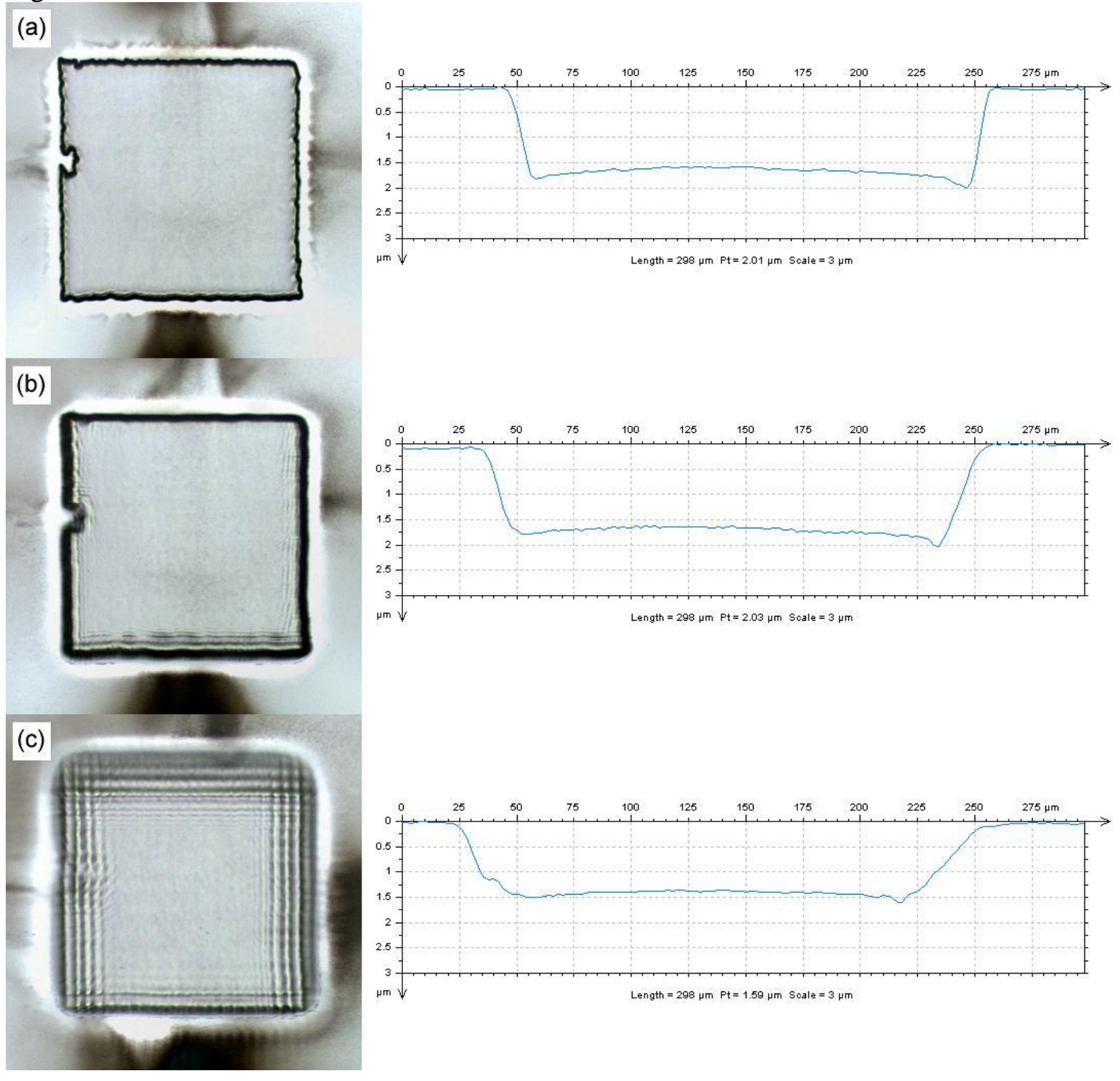


Figure 3:

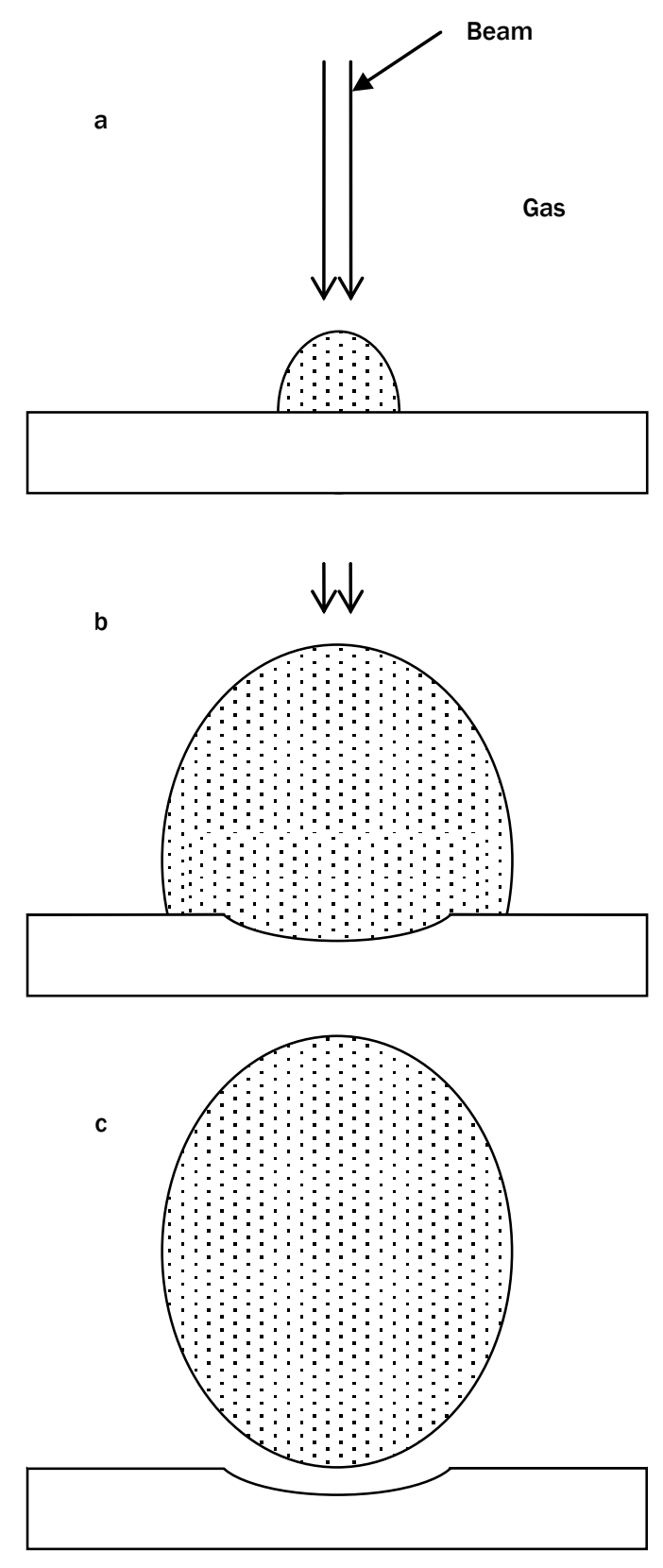


Figure 4:

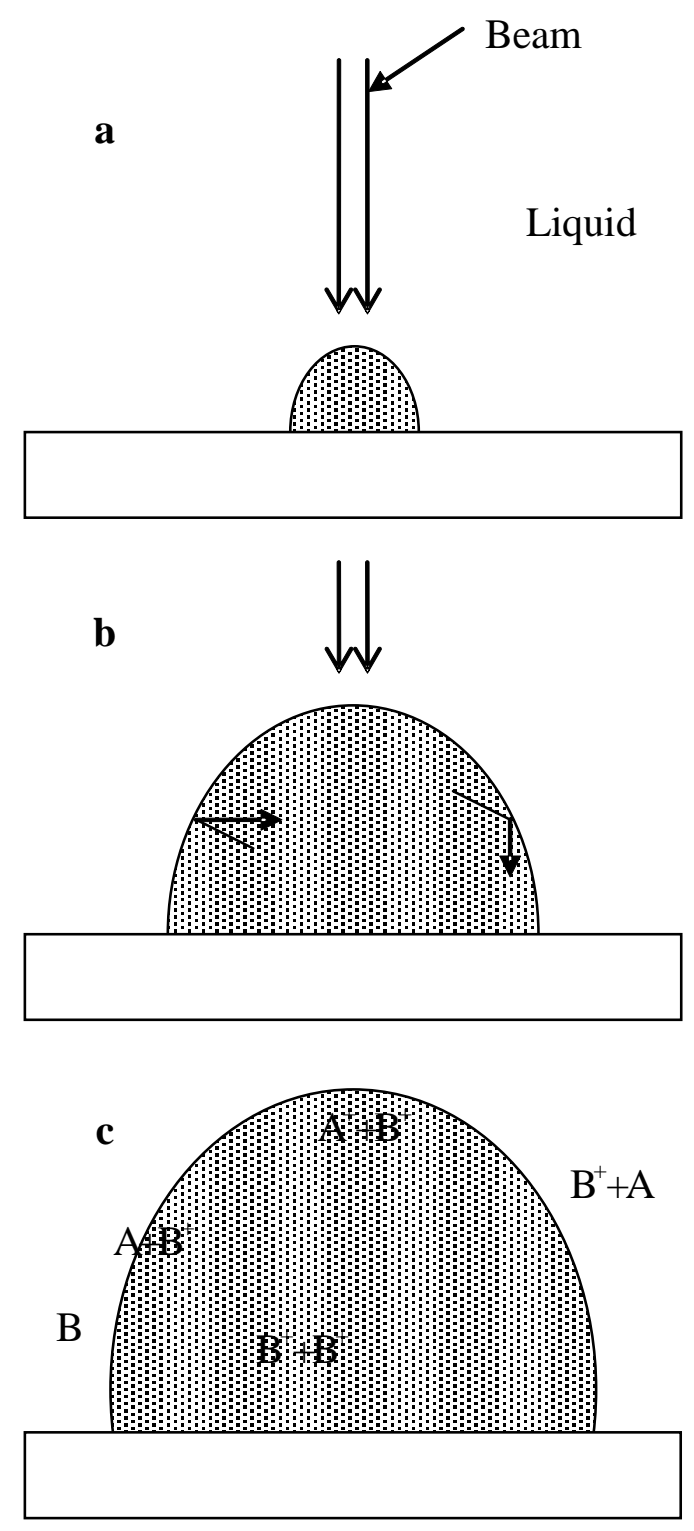


Figure 5:

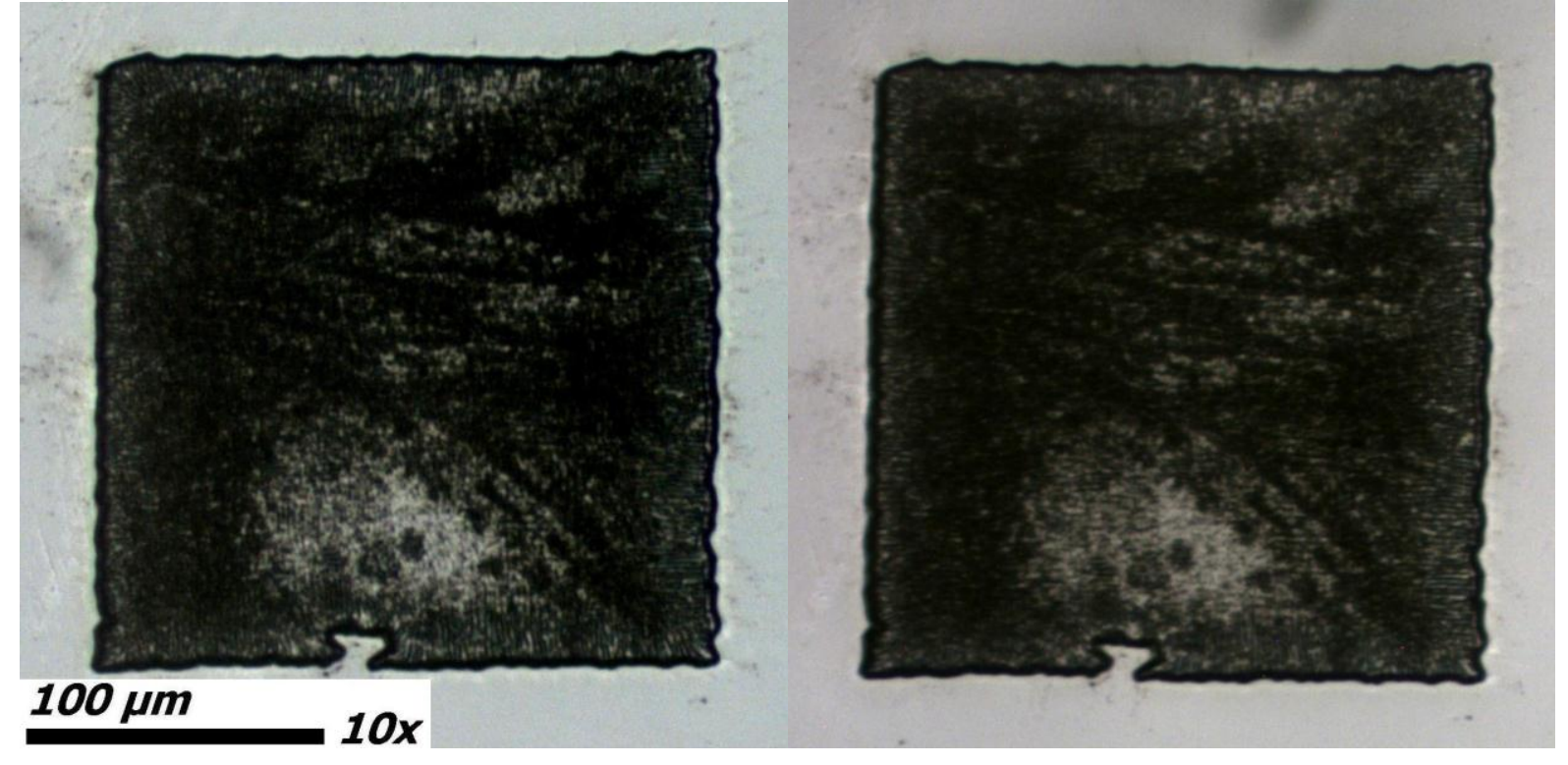


Figure 6:

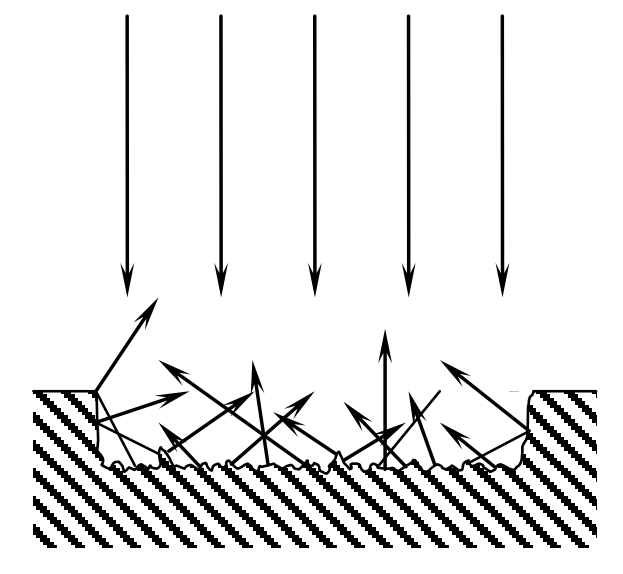


Figure 7:

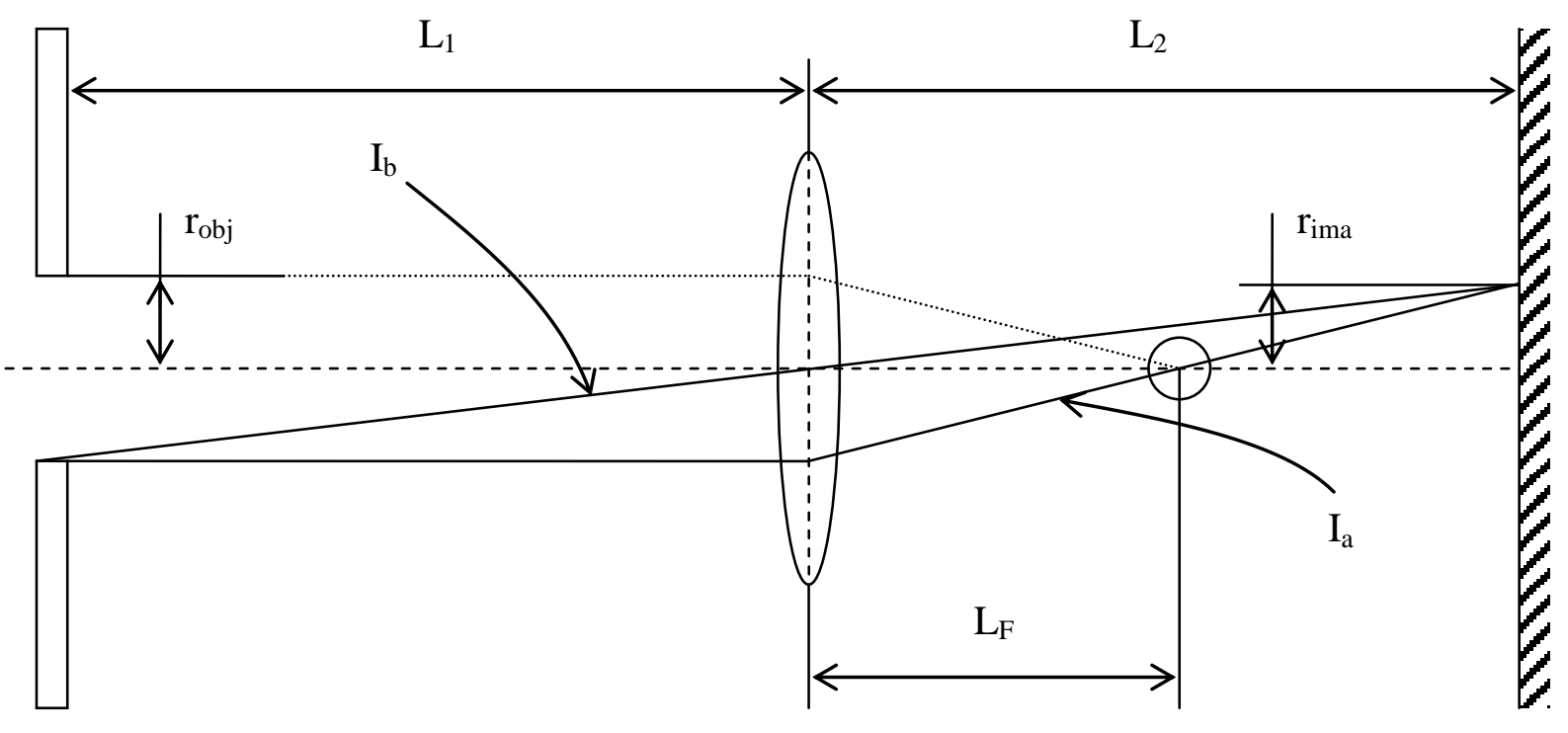


Figure 8:

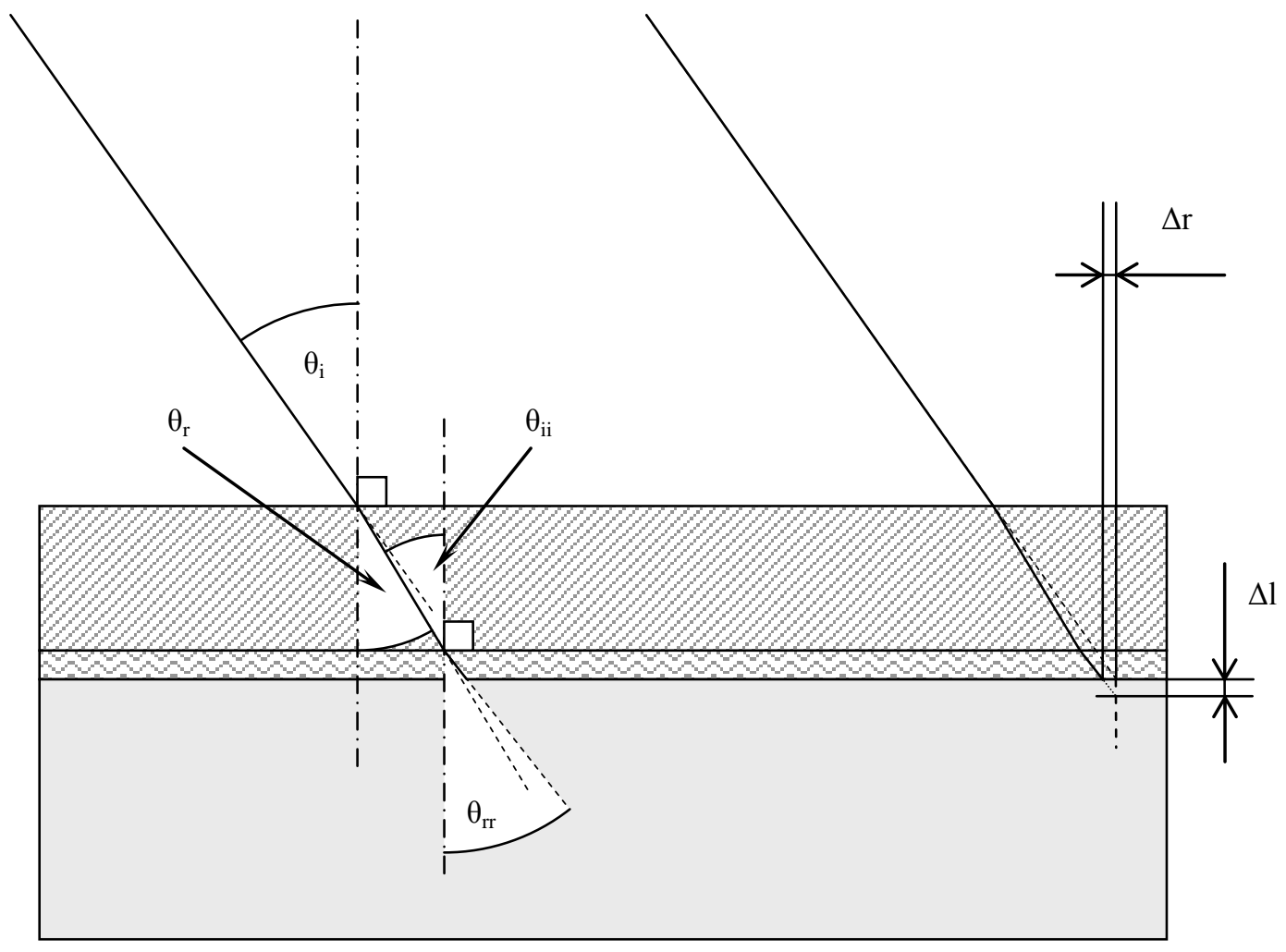


Figure 9:

(a)

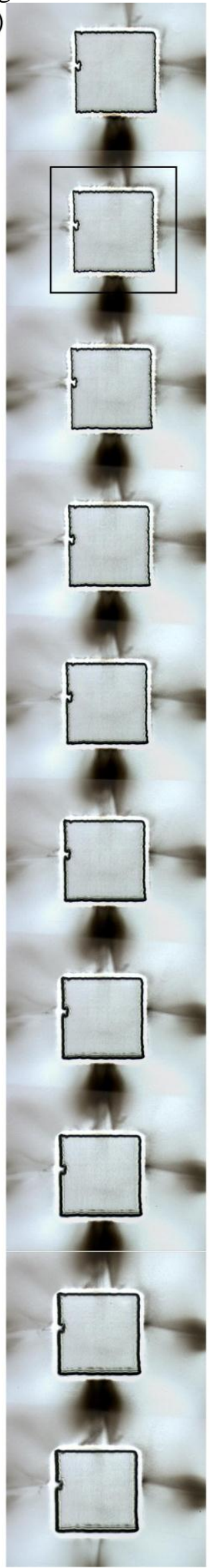

(b)
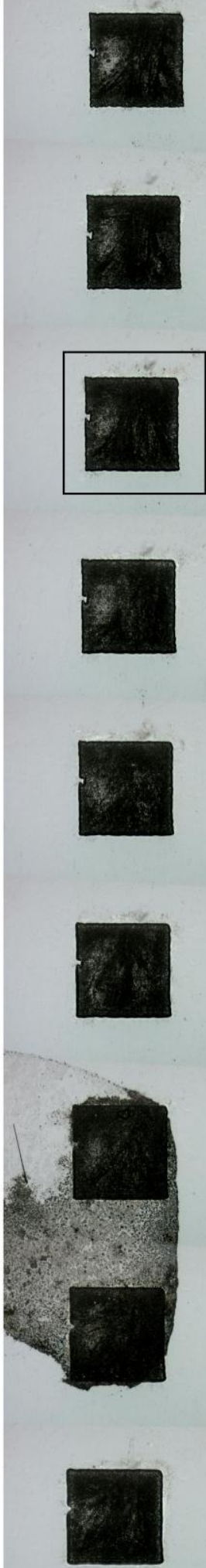
Figure 10:

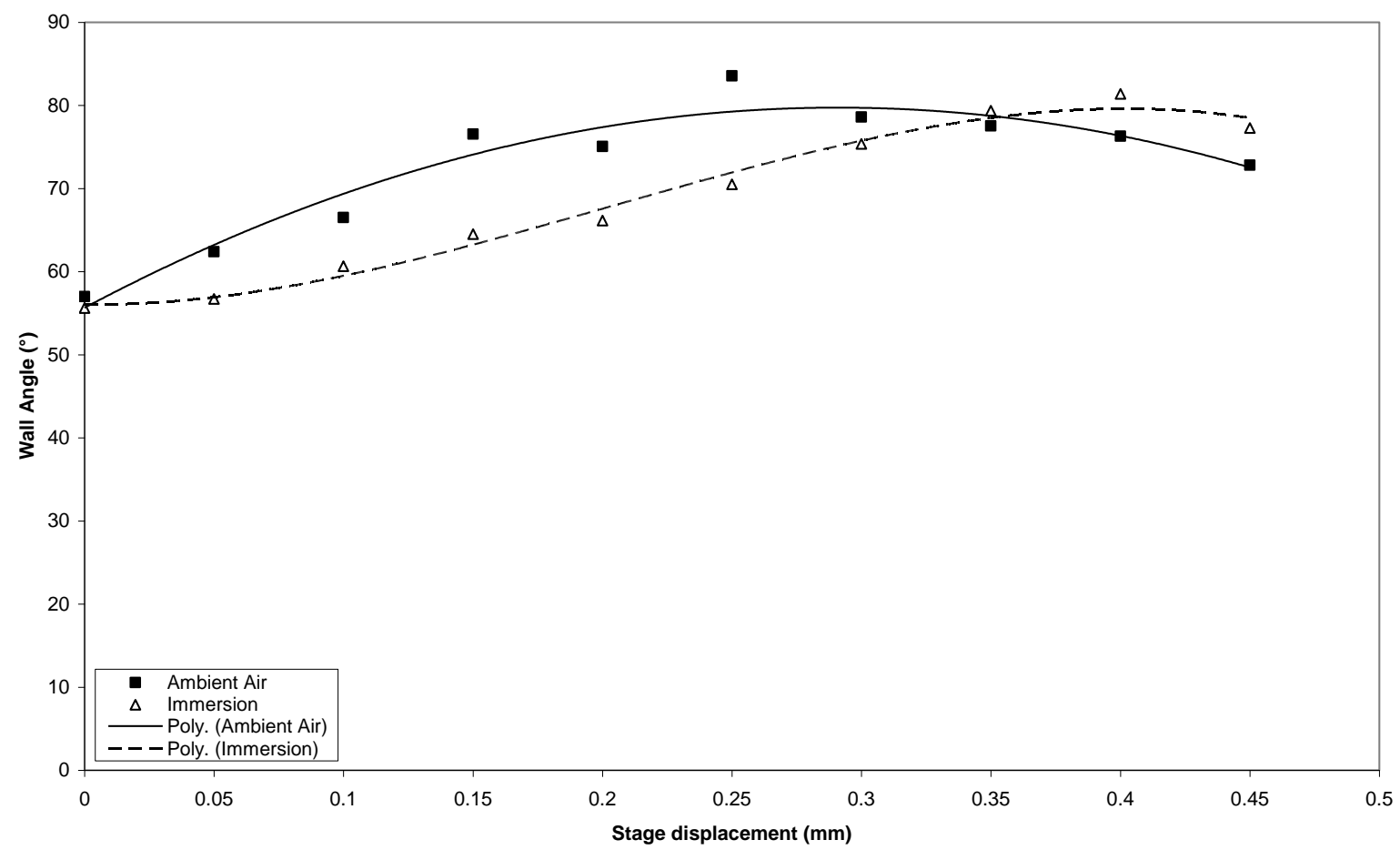

